\title{
Biopolíticas y políticas públicas en sectores poblacionales vulnerables
}

Biopolitics and public policies in vulnerable population sectors

\author{
Alejandro Martín Contino \\ UNR - IUNIR - CONICET \\ martincontino@gmail.com
}

Fecha de recepción

6.2 .14

Fecha de aceptación 10.7.14

\begin{abstract}
Resumen
Se analiza desde el posicionamiento Psicología en Educación, en base al método arqueológico-genealógico, la vinculación existente entre la gubernamentalidad actual, las biopolíticas, y las políticas públicas destinadas a los sectores poblacionales considerados vulnerables por la OMS (niñez, adicciones, sufrimiento mental y discapacidad). Se observan dos clases de biopolíticas: del encierro y de la inclusión. Estas se visibilizan en las políticas públicas a partir de la década de 1980, materializadas en procesos de individuación, concordante con la racionalidad económico-política de la gubernamentalidad actual. La individuación y las políticas del individuo que de ella se desprenden, (que intervienen sobre individuos identificados por nombre y apellido), surgen para solucionar determinadas injusticias sociales, pero demuestran solamente reducir su incidencia y regular su manifestación.
\end{abstract}

Palabras clave: Gubernamentalidad - Biopolíticas - Individuación - Políticas públicas

\section{Abstract}

Through the Psychology in Education approach, based on the archeological-genealogical method, the link between the current governamentality, biopolitics and public policies bound to population sectors which the WHO considered vulnerable is analyzed (childhood, addictions, mental illness and disability). Two kinds of biopolitics 
are deployed: isolation and inclusion, one and the other imprinting public policies since the 1980s through processes of individuation related to the economical-political rationality of the current governamentality. While both individuation and individual policies (characterized by intervening on individuals identified by first and family names), are addressed to solve social injustices, they only attend at reducing their incidence and to regulating their open manifestations.

Key words: governmentality - biopolitics - individuation - public policies

\section{Presentación}

Se analiza en este trabajo el viraje producido en el campo de las políticas públicas destinadas a abordar sectores poblacionales considerados vulnerables por la Organización Mundial de la Salud (OMS, 2010). Es decir, aquellos que se encuentran atravesados por las problemáticas de la vulneración de derechos en la niñez, las adicciones, el sufrimiento mental y la discapacidad.

La vinculación existente entre la gubernamentalidad actual, las biopolíticas, y la racionalidad de las políticas públicas en torno a estos sectores poblacionales es encarada desde la perspectiva crítica conocida como el posicionamiento Psicología en Educación (Emmanuele, 1998), a cuyos efectos se exige un triple descentramiento.

El primero de ellos implica evitar el "institucionalocentrismo" (Foucault, 2009:140). Hay que pasar del interior de la dinámica institucional de cada establecimiento a su exterior, desplazándose de las problemáticas que se manifiestan allí dentro. No hay que perder de vista que cada establecimiento, su existencia, su vigencia y la modalidad de funcionamiento obedece a un ordenamiento social que le es exterior, que lo preexiste, lo excede y lo atraviesa. Dicho ordenamiento se articula a un proyecto gubernamental global que en cada momento histórico se corresponde con una determinada estrategia biopolítica. Si bien al interior de cada espacio se adquieren rasgos específicos, configurando lógicas de funcionamiento que se corresponden con la particularidad de cada problemática, éstas no dejan de remitir a una cierta tecnología de poder que las atraviesa, las condiciona, y llegado el caso, las transforma en algo diferente.

El segundo descentramiento permite dilucidar que la existencia y la vigencia de un espacio concreto - como un Centro de Día para sujetos en situación de discapacidad mental o una Institución de Alojamiento Transitorio para niños vulnerados en sus de- 
rechos- no se explica por el nivel de éxitos o fracasos de su funcionalidad, sino que "se inscribe, de hecho, en estrategias y tácticas que se apoyan incluso en sus propios déficits funcionales" (Foucault, 2009:143). Esto implica que no es pertinente aquí un juicio de valor que señale moralmente un mal funcionamiento en algunos de los establecimientos mencionados, sino que se trata de articular los supuestos fracasos con una racionalidad que le es exterior a él, y que legitima de todos modos la continuidad y la vigencia de su utilización. La cuestión no es entonces por qué tal Centro de Día no logra comúnmente que los sujetos en situación de discapacidad transiten por lo social de un modo diferente a como lo hubiese hecho sin concurrir allí. La cuestión sería más bien, discernir cuál es la funcionalidad política que tiene para la gubernamentalidad actual una modalidad de funcionamiento que no coincida con los objetivos formales pensados para cada espacio. Por último, el tercer descentramiento exige no tomar como punto central de análisis, cualquier objetivación producida por las disciplinas (tales como la discapacidad mental, el sufrimiento mental, la niñez vulnerada en sus derechos o las adicciones), sino el modo en que sus ámbitos de existencia se ven atravesados por ciertas políticas públicas, legislaciones, estrategias terapéuticas, etc. que no pueden mostrarse ajenas a la forma de gubernamentalidad de cada época.

Se propone entonces concebir a cada una de estas problemáticas en términos de territorio (Deleuze \& Guattari, 2002), en tanto su objetivación discursiva configura no sólo una nominación que las identifica, sino también un ámbito específico en el que determinadas reglas se ponen en funcionamiento. En este sentido, "un territorio siempre tiene una zona interior de domicilio o abrigo y una exterior de dominio" (Deleuze \& Guattari, 2002:321).

El territorio remite por otro lado a la realidad vivida, a una realidad afectiva, a un conjunto complejo de registros afectivos que constituyen diferentes formas de vivir en localizaciones determinadas socialmente. Estos registros inciden en el modo de definir la valoración, el placer, los deseo, las emociones. Se trata de un espacio habitado; un espacio en tanto sistema tal como es percibido, tal como es vivenciado. "Establece relaciones complejas entre pertenencia y alienación, identidad e identificación, subjetivación y subjetificación. (...). Define las tonalidades afectivas que dan resonancia y timbre a nuestra vida" (Grossberg, 2012:51). En otras palabras, no es lo mismo para un sujeto ser insertado en un territorio o no estar inserto en él. El modo específico de funcionamiento de un territorio condiciona las formas de subjetividad de los individuos o colectivos que por él se vean atravesados. "El territorio es sinónimo de apropiación, de subjetivación realizada sobre sí mismo” (Deleuze, 2005a:192).

Deviene aquí ineludible marcar un doble aspecto que caracteriza a todo territorio. Éste "no sólo asegura y regula la coexistencia de los miembros de una misma especie, separándolos, sino que también hace posible la coexistencia de un máximo de especies 
diferentes en un mismo medio, especializándolas" (Deleuze \& Guattari, 2002:326). Este punto deviene esencial en el análisis, en tanto es posible vislumbrar el modo en que los territorios de cada problemática social participan de una doble modalidad de funcionamiento. Por un lado, una modalidad que configura una especificidad hacia el interior de sí mismo, y por otro, el atravesamiento de todos ellos por políticas públicas que presentan rasgos en común independientemente de la problemática de la que se trate.

\section{Gubernamentalidad y biopolítica del encierro}

La práctica del encierro como estrategia gubernamental hegemónica frente a las poblaciones que se consideran problemáticas, se generaliza en la época clásica, prolifera en el transcurso de los siglos siguientes, y finalmente se consolida en la Modernidad, en pleno apogeo de las llamadas sociedades disciplinarias (Foucault, 2004).

No solamente los sectores poblacionales que presentan alguna problemática en la circulación y participación social devienen objeto de este tipo de estrategia biopolítica. La población total de las sociedades disciplinarias se ve atravesada por ella, pero en cada sector se presenta bajo una modalidad específica. Es decir, la estrategia biopolítica del encierro se sostiene a lo largo de varios siglos, adquiriendo matices diferentes de acuerdo a las características de cada sector poblacional. Se caracteriza por un proyecto basado en espacios relativa o completamente cerrados y con sistemas disciplinarios absolutamente normativizados hacia el interior de cada uno de ellos.

Estos lugares que se distinguen de los otros, que son radicalmente distintos, por encargarse de alojar en su interior sujetos que son considerados diferentes, que están desviados, que funcionan disfuncionalmente, pueden denominarse heterotopía (Foucault, 2010). Las heterotopías cuentan con la asombrosa posibilidad de yuxtaponer en un mismo lugar real, espacios que en otras circunstancias son incompatibles entre sí, o que tal vez puedan sostenerse operando juntos durante algún tiempo, pero en otro devengan nuevamente incompatibles.

En ocasiones, conforman circuitos específicos frente a los cuales no siempre y no cualquiera puede elegir no transitarlos: "el individuo no deja de pasar de un espacio cerrado a otro, cada uno con sus leyes" (Deleuze, 2005b:115).

Ahora bien, existen espacios de encierro que no están destinados a la totalidad de la población, sino a quienes presentan alguna dificultad para transitar por lo social de una manera esperable de acuerdo a la racionalidad económica-política del capitalismo industrial naciente. Esto conforma "una lógica propia de la modernidad, la lógica binaria, de la inclusión-exclusión. Los que circulan y los que son encerrados, los que ejercen sus derechos y los que no tienen voz para exigir por los suyos” (Belgich, 2004:51). Es 
decir, la sociedad disciplinaria se divide en una parte de la población que circula por el espacio social común sin mayores dificultades, y otra parte que se ve retenida en espacios conformados de acuerdo a lógicas de funcionamiento radicalmente diferente a las del espacio público.

De este modo, entre el siglo XVII y principios del XX las heterotopías destinadas a sectores poblacionales que representan una problemática, se configuran bajo la modalidad de espacios de características asilares, comenzando a surgir simultáneamente en varios países de Europa (Fernández Bruno \& Gabay, 2003).

El Hospicio -tal es el nombre que recibe- está conformado en base a una gran estructura edilicia cuyo ingreso y egreso se encuentra fuertemente controlado. Se corresponde a su vez con aquello que Goffman (1970) define como instituciones totales. Se trata de lugares de confinamiento donde viven individuos obligados a seguir una rutina de vida formalizada, bajo el control y dirección de un órgano burocrático y que se caracterizan por contar con un contacto limitado con el resto de la sociedad.

En su interior, enviados por diversas instancias estatales de orden jurídico, reúnen a una serie de individuos de rasgos muy diversos entre sí (prostitutas, vagos, delincuentes, locos, etc.). Es decir que uno de sus rasgos característicos es el de no diferenciar a quienes allí se interna, por no existir un proyecto terapéutico rehabilitatorio (Foucault, 2008a), sino solamente un proyecto político de invisibilizar de la circulación social este tipo de sectores poblacionales.

A fines del siglo XVII y comienzos del XVIII factores médicos, académicos y filosóficos enmarcados en el Iluminismo, modifican la orientación de la psiquiatría, con el tratamiento moral de Philippe Pinel, quien transforma determinadas prácticas institucionalizadas en los hospicios (tales como mantener a los alienados encadenados), orientado el funcionamiento de los asilos hacia una dirección que posibilitara algo del orden de la rehabilitación, por dirigirse no al cuerpo sino al alma de los internos (Galende, 2006; Foucault, 2008b). De hecho, uno de los actos más relevantes en este momento de virajes, es la liberación de las cadenas que sujetan a 3 ó 4 decenas de individuos que se hallan en dos de los hospitales que quedan bajo su égida (Galende, 2006). Este acontecimiento tiene lugar en 1793, fecha que puede ser considerada por estas razones como el nacimiento del alienismo ${ }^{2}$. Del mismo modo, propone prescindir del recurso de la inmersión en agua como forma de tratamiento de la alienación. De hecho, se refiere a ello como un delirio de los médicos, peor que el de los enfermos.

Kraepelin, por su parte, luego de realizar un relevamiento del funcionamiento de los asilos en Alemania, denuncia que en ellos no se puede distinguir y diferenciar entre sí claramente los medios de castigo -dirigidos a obtener disciplina y sometimiento del en- 
fermo en el interior del establecimiento-, de las medidas terapéuticas (Galende, 2006). Principalmente debido a que en general se trata de la misma técnica, cuya supuesta finalidad es doble, tal como sostiene el director del asilo de Stralsunde, al afirmar que "algunos azotes con vara de abedul obran verdaderos milagros" (Kraepelin, 1999, según cita Galende, 2006:57).

De esta manera, se posibilita la individualización de algunos pacientes, que pese a sus síntomas pueden volver a vivir en la comunidad reintegrados a su familia. Hasta este momento, en que se puede considerar que surge la medicina moderna, nadie sale del Hospital; la técnica médica de estos siglos no permite al individuo hospitalizado abandonar dicho espacio con vida (Foucault, 1998).

Diferentes discursos constituyen el entramado necesario para la emergencia de los espacios de encierro, pero desde el momento en que éstos comienzan a emerger, uno de ellos presenta una predominancia radical: el discurso religioso. Se encuentra presente desde los orígenes de los establecimientos destinados a alojar grupos de individuos que hay que proteger, cuidar, reformar o corregir. Según afirma Foucault, "en la mayoría de los casos eran las órdenes religiosas las que tenían, si no la iniciativa, sí al menos la responsabilidad de la gestión de estos establecimientos" (Foucault, 2008b:92). De hecho, la nominación de tratamiento moral propuesta por Pinel para definir un modo de tratamiento que se dirija al alma y no al cuerpo, presenta ya en sí mismo un fragmento de materialidad discursiva del discurso religioso.

Sin embargo, esta predominancia de la institución Iglesia encuentra una situación de confrontación en los intentos expansionistas del discurso médico, legitimado socialmente a partir de la pretensión de cientificidad que en un determinado momento histórico lo atraviesa. De este modo, los espacios asilares se ven cada vez más profesionalizados y medicalizados en los abordajes desplegados en su interior.

Cabe resaltar que de acuerdo al pensamiento foucaultiano, no existe sociedad que no cuente con sus propias heterotopías, a pesar de que éstas puedan variar de una sociedad a otra, o bien de un momento de una sociedad a otro. Se observa en este viraje entonces, el modo en que una heterotopía muta, se transforma en mayor o menor medida, reacomodándose a un nuevo ordenamiento discursivo y de poder, en cuya trama se insertan nuevas objetivaciones.

Así las cosas, las figuras del médico alienista (luego devenido psiquiatra), el enfermero, etc., van proliferando a partir de la extracción de saber que deviene posible en virtud de las estrategias de poder que mantienen internados a todo un conjunto de individuos que se caracterizan por pensarse como acontratables (Belgich, 2004). 
A pesar de ello, la creciente medicalización de estos espacios coexiste con la impronta de otras instancias tales como las Sociedades de Beneficencia, las Damas de Caridad, etc., las cuales se mantienen a cargo de ellos o incluso en algunos casos, son responsables de su fundación. De todos modos, si bien éstas no son completamente dependientes de la institución Iglesia, sí sostienen una fuerte raigambre en el discurso religioso.

Aproximadamente hacia fines del siglo XIX y principios del siglo XX (aunque en Europa se produce con algo de anterioridad), estos espacios asilares se ven a su vez fuertemente atravesados por los lineamientos gubernamentales pertenecientes a lo estatal, logrando desplazar gradualmente tales sociedades.

Se produce de este modo un viraje desde la figura del hospicio hacia la del hospital general, y de ésta hacia la especificidad de diversas heterotopías, entre las cuales se encuentran los hospitales o asilos de alienados, los hogares de huérfanos o expósitos, etc. Por lo tanto, los espacios asilares de encierro destinados a sectores poblacionales problemáticos, no siempre portan o mantienen las mismas características ni modalidades de funcionamiento.

\section{Heterotopías del encierro y normalización en la región de Rosario (Argentina)}

Extendiendo sin demasiadas modificaciones esta estrategia biopolítica heredada de Europa, los establecimientos asilares surgieron en esta región en la época de la Colonia. Se llevaba a los locos alborotados a la cárcel del Cabildo o a los denominados "Cuadros de Dementes" de los hospitales, en los que éstos "vegetaban sin ninguna clase de tratamiento especial" (Fernández Bruno \& Gabay, 2003:17).

Y siguiendo el mismo recorrido observado en Europa, tanto la medicalización como el entrecruzamiento con el Estado comienzan a posibilitar también esta región geográfica la sustitución de los grandes Hospicios por una serie de Asilos de Alienados, ya sea en localidades pequeñas, o bien determinadas zonas ubicadas en los márgenes de algunas grandes ciudades.

Un recorrido por la construcción de heterotopías destinadas al abordaje de problemáticas vinculadas a ciertos sectores poblacionales en la región de Rosario, muestra que el hoy denominado Centro Regional de Salud Mental "Dr. Agudo Avila" se funda como Hospital de Alienados el 2 de octubre de 1927 en un sitio geográfico muy particular, que coincide con otras heterotopías destinadas a grupos poblaciones que también requieren ser abordados. En la misma calle (Suipacha) se encuentran emplazados aún hoy dos establecimientos emblemáticos: el ex hospital psiquiátrico y la actual Unidad Penitenciaria de Detención $N^{\circ} 3$. 
Se elige dicha zona dado que en la época del centenario de la declaración de la Independencia (momento en el que surgen este tipo de espacios en la ciudad de Rosario), ésta se caracteriza por la presencia de muelles, prostíbulos, bodegones; habitada por rufianes, prostitutas, obreros, estibadores, mafiosos, etc. En suma, individuos considerados marginales.

Por su parte, el actual Hospital Carrasco (uno de los más antiguos de la ciudad, con 115 años de existencia), es ubicado en la misma zona, creado prioritariamente para tratar enfermedades infectocontagiosas muy graves para la época, tales como la lepra, la peste bubónica, el tifus, el cólera, la viruela, la difteria y la tuberculosis. En sus comienzos había salas organizadas en su interior a la manera de los pabellones y otras que se ubican por debajo del nivel del suelo (cuyos ventanales respiraderos pueden ser observados actualmente en el sector del patio). De este modo, se logra aislar no solo a los contagiados del resto de la población de la ciudad, sino que también se logra un aislamiento y una separación entre las distintas clases de enfermos.

En este caso, la razón de su ubicación en lo que en ese momento es el extremo oeste de la ciudad, es que al ser infrecuentes los vientos desde dicho punto cardinal, se ve reducida la probabilidad de que este fenómeno climático trasladara las enfermedades contagiosas hacia la zona más habitada del centro de la ciudad.

No es de extrañar por lo tanto, que la primera nominación que dicho Hospital recibe es la de "Casa de aislamiento" (en 1914 se le cambió el nombre por el de Hospital “Intendente Gabriel Carrasco"). Las reseñas de la época refieren a que la nominación que la población le otorga es la de "Lugar del buen morir", o "Casa de los lamentos" lo cual parece dar cuenta de una priorización de fines no ya de encierro, pero sí de aislamiento, que se montan sobre los terapéuticos.

El ideal normalizador deviene liminar en una sociedad caracterizada por tecnologías de poder disciplinarias. La utopía que postula la factibilidad del desarrollo de estrategias, tácticas, técnicas, saberes y conocimientos que logren reducir la brecha entre lo anormal y lo normal legitima el surgimiento de toda una multiplicidad de espacios radicalmente diferentes a otros espacios sociales con los que sin embargo coexisten. El aislamiento deviene así un recurso estratégico imprescindible en esta racionalidad normalizadora inherente a una sociedad que se muestra cada vez más medicalizada.

La medicalización del sufrimiento va seguida de una cierta naturalización de la enfermedad mental como una entidad en sí misma (...). No son sino los discursos en tanto prácticas positivas los que hacen aparecer a los fenómenos, y no los fenómenos los que hacen aparecer los discursos (Albano, 2006:12). 
Es decir que la medicalización de la sociedad legitima una lectura de ciertos fenómenos presentes en lo social siempre bajo la perspectiva de que se está frente una patología individual. "En síntesis, patologización para justificar la medicalización, medicalización para justificar la institucionalización” (Contino, 2010:170). Especialmente en lo concerniente a toda aquella problemática presente en lo social.Y ello habilita a determinadas estrategias y prácticas y no a otras, casi siempre caracterizadas por la circunscripción de conjuntos de individuos incluidos en cada categoría, en determinados circuitos que funcionan de manera exclusiva para sujetos atravesados por la misma cuestión.

Se institucionaliza entonces la lógica del encierro y el aislamiento como respuesta biopolítica frente a lo irreductible de la sociedad. Dan cuenta de ello el ya mencionado lugar del buen morir, o bien la Colonia de Oliveros, situada en la localidad santafesina de la que recibe el nombre, en donde son enviados a principios del siglo XX los alienados considerados incurables, para que pasen allí el resto de su vida.

Circuitos especiales organizados para determinados individuos que son dirigidos mediante un mecanismo de poder completamente legitimado en lo social. Pero ello conlleva una suerte de amenaza psiquiátrica.

Si no eres como todo el mundo, eres anormal; si eres anormal, estás enfermo. Estas tres categorías: no ser como todo el mundo, ser anormal, y estar enfermo, pese a ser muy diferentes, se han encontrado asimiladas las unas a las otras (Droit, 2008:76).

Cabe preguntarse: los establecimientos que existen en este tipo de territorios, ¿continúan siendo en la actualidad tributarios de la racionalidad propia del abordaje institucional de las estrategias biopolíticas del encierro? ¿Siguen siendo elegidos para su emplazamiento físico los márgenes de la sociedad urbanizada? ¿Continúan las estructuras edilicias caracterizándose por grandes dimensiones, por un interior bien diferenciado del exterior, por los rasgos asilares de su circulación interna? ¿Permanece activo un modo de gubernamentalidad que determina al interior de estos espacios otros, una modalidad de distribución y funcionamiento de las redes del poder acorde al que puede observarse cuando se ejerce "el poder psiquiátrico en estado puro, casi sin reelaboración alguna”, por utilizar la expresión de Foucault (Foucault, 2008b:251)?

En las secciones siguientes se proponen respuestas a estas interrogantes. 


\section{El viraje biopolítico. De la marginalidad y el aislamiento de los espacios de encierro a la inclusión y la visibilidad de los dispositivos de seguridad}

En la época de las biopolíticas del encierro, hay mecanismos vigentes que en razón de la gubernamentalidad y del suelo histórico político de la época, no generan un rechazo social significativo ni son objeto de fuertes críticas. Es así que en los siglos XIX y principios del XX, el aislamiento, el encierro, la reclusión, la invisibilización, la segregación física a otros espacios diferentes a aquellos por donde circula el resto de la población, no sólo no es cuestionado, sino que incluso es demandada desde el mismo tejido social. Ahora bien, actualmente la sociedad se encuentra "en una crisis generalizada de todos los lugares de encierro" (Deleuze, 2005b:115). Las estrategias biopolíticas que utilizan esencialmente el aislamiento y/o el encierro para con los desviados respecto de la normatividad de la época se ven transformadas -en especial, durante las últimas tres o cuatro décadas-, favoreciendo la proliferación de una multiplicidad de mecanismos y tácticas que se presentan como novedosas para el abordaje de los fenómenos humanos problemáticos que se manifiestan en lo social. Sin embargo, ello no exime a que en ocasiones se lleven a cabo en los mismos establecimientos o espacios en que funcionaba la lógica del encierro.

Cada territorio que representa una problemática para el Estado, presenta mecanismos que en la actualidad contemplan acciones tendientes a favorecer o posibilitar la inclusión social de todos estos sectores de la población que anteriormente se encontraba fuera del campo de visión, circulación y participación social. La inclusión, en tanto estrategia biopolítica emergida entre mediados y fines del siglo XX, remite a la necesidad filosófica, moral, pedagógica, económica, política y social de aceptar y valorar las diferencias, favoreciendo el acceso de ciertos sectores de la población a un modo de transitar lo social que en principio, habilite el acceso a las mismas oportunidades de las que puede disponer el resto de la población.

Por lo tanto, la cuestión de la inclusión parece atravesar cada uno de los territorios que se configuran a partir de la objetivación de cada sector poblacional específico. Una racionalidad diferente a la de la época del encierro los penetra y los transforma, producto de la elaboración de nuevas tecnologías de poder y entramados discursivos diferentes, en tanto preexiste y excede a cualquiera de esos territorios.

Ahora, cabe advertir que el surgimiento de esta nueva racionalidad biopolítica aporta una lógica específica para pensar las políticas públicas, las legislaciones, las prácticas, las disposiciones edilicias, las enunciaciones, etc. Y esta nueva lógica se encuentra directamente vinculada a las demandas y los reclamos sociales que emergen en cada uno de estos territorios. 
Dichos reclamos se corresponden en primer lugar, con los efectos jurídicos producidos en la mitad del siglo XX con la Declaración Universal de los Derechos Humanos, en diversos territorios de diferentes sectores de la población (las mujeres, los minusválidos, los enfermos mentales, los homosexuales, los menores, etc., tal como los nominan las objetivaciones de la época), que comienzan a denunciar la situación de exclusión, marginación, discriminación y opresión que vivencian cotidianamente. Dicha Declaración funciona entonces como una herramienta jurídica trascendental para que estos sectores poblacionales que quedan configurados como problemáticos, patológicos o simplemente como necesitados de tutelaje, puedan comenzar a intentar transformar -reterritorializar- el territorio en el que fisica y subjetivamente se encuentran.

Las políticas públicas actuales ya no contemplan las tácticas del encierro y del aislamiento para las intervenciones dirigidas a sectores de la población en los que se materializa alguna dificultad para transitar por lo social. Por el contrario, la regulación y el control de la circulación y la participación social se muestran como las estrategias prioritarias en relación a estos sectores poblacionales. Al decir de Deleuze, "son las sociedades de control las que están reemplazando a las sociedades disciplinarias” (Deleuze, 2005b:116). Estos espacios de las sociedades de control comienzan a configurar heterotopías diferentes a las ya existentes en las sociedades disciplinarias. Éstas presentan tres coordenadas principales que orientan su funcionamiento.

En primer lugar, estos espacios funcionan exclusivamente en relación a ciertos fenómenos sociales que representan una problemática específica que -de acuerdo a la gubernamentalidad actual- requiere ser abordada por el Estado. Dichos fenómenos -retraso mental, vulneración de derechos en la niñez, adicciones, padecimiento mental, etc.-, no son deseables, no son buscados, pero se presupone que ocurrirán y el Estado debe estar en condiciones de responder frente a su manifestación. En otras palabras, "se inserta el fenómeno en cuestión dentro de una serie de acontecimientos probables" (Foucault, 2009:21).

En segunda instancia, se observa que las tácticas implementadas se encuentran absolutamente condicionadas por la cuestión presupuestaria. De este modo, toda una serie de establecimientos -tales como las instituciones de alojamiento transitorio-comúnmente denominadas hogares- para la niñez en situación de vulneración de sus derechos, las granjas o comunidades terapéuticas para adicciones, los centros de salud mental para los casos de sufrimiento mental, etc.- representan un costo excesivo, tanto en lo que respecta a la puesta a punto de una estructura edilicia, como a lo que concierne al personal que queda a su cargo.

En razón de ello, se vienen implementando dos medidas que suelen repetirse en la mayoría de los territorios: desde la década de 1990 se delega en las obras sociales la co- 
bertura de todo lo necesario en el plano terapéutico, y se habilita al sector privado a la construcción de espacios destinados a su abordaje (aun cuando éstos funcionen bajo la lógica empresarial de la rentabilidad económica, inherente al sistema capitalista). Existe siempre la opción de lo público, pero los obstáculos de todo funcionamiento estatal a causa principalmente de lo limitado del presupuesto, restringe su acceso a un número muy limitado de sujetos. En otras palabras, "las reacciones del poder frente a este fenómeno se incorporarán a un cálculo de costos” (Foucault, 2009:21).

Y la tercera y última coordenada que orienta el funcionamiento de este tipo de espacios remite al porcentaje de lo tolerable en cuanto al índice de incidencia de estos fenómenos. No es preocupante que exista un caso de un niño violentado, abusado o abandonado. Deviene una preocupación cuando los casos de niños en situación de vulneración alcanzan un cierto porcentaje de la población. No es preocupante que un individuo no logre insertarse socialmente bajo un modo de circulación y participación social acorde a la del resto de la población. La preocupación aparece cuando esto le ocurre a todo un sector poblacional. Es decir, estos espacios, "en lugar de establecer una división binaria entre lo permitido y lo vedado, se fijarán por una parte una media considerada como óptima y por otra límites de lo aceptable, más allá de los cuales no habrá que pasar' (Foucault, 2009:21).

Ahora bien, estas tres coordenadas que describen el modo en que se conciben ciertos fenómenos - como acontecimientos probables, incorporados a un cálculo de costos y atravesados por límites óptimos y máximos-, son los aspectos básicos que caracterizan el funcionamiento de aquello que Foucault (2009) denomina dispositivos de seguridad.

Los dispositivos de seguridad constituyen espacios que se caracterizan por ejercer sus tecnologías de poder no tanto sobre el cuerpo de los individuos (como ocurre en los espacios disciplinarios), sino más bien sobre el conjunto de una población. No se busca tanto una posición subjetiva dócil y útil como la ganancia de una cierta seguridad en relación a algún riesgo.

No se trata en absoluto de construir dentro de un espacio vacío o vaciado, tal como sucedía en las sociedades disciplinarias. (...) Se trata simplemente de maximizar los elementos positivos, que se circule lo mejor posible, y minimizar al contrario, los aspectos riesgosos o inconvenientes (...) que jamás se los suprimirá del todo (Foucault, 2009:39). 
Ahora, si ese riesgo jamás podrá ser eliminado del todo, el modo en que se configuran las intervenciones en este tipo de espacios es a través del cálculo de probabilidades, intentando incidir en series abiertas de elementos que circulan, que se desplazan, que se acumulan, que escasean. Por lo tanto, el medio que se configura a partir de los dispositivos de seguridad incluye lo polivalente, lo transformable, lo aleatorio, la temporalidad. A diferencia de ello, en los espacios disciplinarios hay celdas especificadas, temporalidades cerradas, funciones prefijadas, ordenamientos funcionales, vigilancia y examen que no dejan nada librado al azar.

A su vez, los dispositivos de seguridad tienden a ampliarse, mostrándose siempre predispuestos a integrar permanentemente nuevos elementos. Tanto en lo que respecta a la articulación entre diferentes espacios, como hacia su propio interior.

De este modo, las instituciones de alojamiento transitorio por ejemplo, suelen funcionar de manera articulada a redes distritales que las vinculan a centros de salud, hospitales, centros de acción familiar, escuelas comunes y especiales, etc. El Centro Regional de Salud Mental Dr. Agudo Ávila, para sujetos en situación de padecimiento mental, por su parte, suele sostener lazos con establecimientos de diversa naturaleza.

A su vez, hacia el interior de los mismos espacios, puede encontrarse un conjunto de estudiantes realizando prácticas, pasantes, residentes, colaboradores, etc. En los Centros de Día para sujetos en situación de discapacidad mental, suelen trabajar algunas horas a la semana de manera ad honorem profesionales recién recibidos, de modo de facilitar la adquisición de una cierta experiencia profesional, a la vez que se obtiene mano de obra gratuita. También es común encontrar actividades recreativas o culturales que se presentan de manera voluntaria y solidaria, tales como obras de teatro, conciertos de tango por parte de un cantante de la ciudad, etc.

A su vez, en tanto se pretende regular la circulación, ninguno de estos establecimientos funciona bajo el régimen de puertas cerradas, con internación total por tiempo indeterminado y bajo decisiones arbitrarias centralizadas en una figura concreta -Juez de Menores; Director de Hospital de Alienados, etc.-, tal como ocurre en los establecimientos que habilita las biopolíticas del encierro. El Marco Básico de Funcionamiento de las Instituciones en Discapacidad por ejemplo, sostiene al respecto que "deberán privilegiarse las pequeñas instituciones, sobre las grandes, que difícilmente puedan cumplir estos objetivos y permitir a las personas con discapacidad el goce de condiciones de vida más dignas".

Es decir, los actuales espacios se consideran siempre lugares de tránsito, aunque luego este tránsito encuentre un "cuello de botella", un obstáculo o una imposibilidad (por 
falta de otro Centro de Día o Centro Educativo Terapéutico que ofrezca mejores condiciones de tratamiento; por falta de ofrecimiento de empresas para contratar a quienes logran formarse en los Talleres Protegidos; por falta de individuos, familias o parejas que decidan adoptar niños en condiciones de adoptabilidad; etc.).

A diferencia de los espacios disciplinarios que funcionan bajo la lógica dicotómica de lo prohibido y lo obligatorio, los dispositivos de seguridad ya no buscan la manifestación cero de un acontecimiento. No busca anularla ni frenarla, sino limitarla y regularla. El mecanismo de encauzamiento de las conductas no sigue siendo entonces la sanción normalizadora -destinada a evitar que el acontecimiento problemático se repita-, sino la contención del individuo para que se reduzca al mínimo la probabilidad de su aparición, pero siempre considerando la posibilidad de que en cualquier momento puede volver a darse dicha situación.

La disciplina delimita explícitamente entre lo normal y lo anormal; clasifica elementos de acuerdo a objetivos determinados; secuencia y coordina de la mejor manera posible; y llegado el caso, modifica individuos, tiempos, gestos, actos, operaciones, etc. Se trata más de una normación que de una normalización. Ahora bien, un dispositivo de seguridad sólo puede funcionar si le es concomitante una apertura tal que permita la libertad. Debe existir la posibilidad de movimientos, desplazamientos, circulación (tanto de gente como de cosas).

De todos modos, cabe resaltar que no se trata de la desaparición de la lógica binaria de lo normal y lo anormal, instauradora de todo tipo de dicotomías a partir del funcionamiento de las prácticas discursivas, sino de otro modo de tratamiento de lo anormal:

Ahora habrá un señalamiento de lo normal y lo anormal, un señalamiento de las diferentes curvas de normalidad, $y$ la operación de normalización consistirá en hacer interactuar esas diferentes atribuciones de normalidad y procurar que las más desfavorables se asimilen a las más favorables. (...) Por consiguiente, yo diría que ya no se trata de una normación sino más bien, o en sentido estricto, de una normalización (Foucault, 2009:83).

De esta manera, si las tecnologías de poder propias de los dispositivos de seguridad muestran su funcionamiento en la regulación de la circulación, más que en la desaparición o la evitación de un fenómeno, la cuestión de la circulación deviene central. Por lo tanto, las tácticas y los mecanismos tendientes a lograr la circulación, la participación y la inclusión, devienen prioritarios. Los sujetos en situación de discapacidad mental ya no pueden permanecer encerrados en una habitación de su casa o aislado en un 
establecimiento de alojamiento permanente. Los pases libres de transporte público; los automóviles con franquicia; los transportes especiales; los espacios y estacionamientos reservados; la accesibilidad fisica, edilicia, y de internet; el cupo laboral obligatorio; la cobertura total de todo tratamiento vinculado a la problemática; etc., se instituyen como tácticas fundamentales para favorecer la circulación y participación social de los sujetos en situación de discapacidad.

Asimismo, la ley provincial 12.967 de protección integral de los derechos de niños, niñas y adolescentes, de 2010, prohíbe que el Estado tome a su cargo a un niño alojándolo por fuera de su familia de origen por un período de tiempo superior al año y medio, ya sea en instituciones de alojamiento transitorio, en la familia biológica ampliada, o bien en una Familia Solidaria. A diferencia de ello, en la época del Patronato se mantenía a los menores marginados del espacio social hasta su mayoría de edad, en ámbitos asilares tales como el Hogar del Huérfano. Por su parte, la sustitución de grados radiales en Hogares en favor del cursado escolar en los establecimientos correspondientes, así como la prioridad que se observa en cuanto a las opciones de la familia ampliada o la familia solidaria en detrimento de las instituciones de alojamiento transitorio, se presenta en el territorio de la niñez como ejemplos de tácticas destinadas a favorecer la circulación social.

Por otro lado, el trabajo desde lógicas desmanicomializadoras (Cohen \& Natella, 2007) sostenido desde hace varios años en el Centro Regional de Salud Mental estatal viene reduciendo drásticamente el número de sujetos internados en su interior. A su vez, desde la reglamentación de la nueva ley de Salud Mental (ley 26.657 de 2010), la creación de nuevos manicomios o las internaciones arbitrarias se ven finalmente impedidas.

\section{Políticas públicas en la época de las biopolíticas de la inclusión}

Ahora bien, la cuestión de la circulación social remite también a las políticas públicas que regulan su favorecimiento, aunque siempre estén en relación a las biopolíticas de inclusión.

La protección y la promoción de derechos de ciertos sectores poblacionales impregnan la situación actual de los diversos territorios considerados vulnerables. "Se trata de poner de relieve cierto nivel en que la acción de quienes gobiernan es necesaria y suficiente" (Foucault, 2009:87).

La racionalidad inherente a las biopolíticas del encierro a fines del siglo XIX y principios del siglo XX recorre los territorios de esas objetivaciones psiquiátricas que son la debilidad mental, la infancia, la alienación, las toxicomanías, etc. Dicho atravesamiento posibilita que se objetiven estos fenómenos en dicho momento histórico de un modo 
análogo y complementario, compartiendo espacios y tácticas. Esto también implica la existencia de otros espacios -heterotopías- específicas para cada fenómeno, como así también un entrecruzamiento discursivo y de tecnologías de poder -tanto disciplinarias como biopolíticas- inherentes a este modelo, que legitima socialmente las construcciones discursivas y las prácticas en dicha época.

Ahora bien, a partir de la segunda mitad del siglo XX se producen múltiples virajes discursivos y de poder que contribuyen a una reformulación de aquello que hasta este momento se viene sosteniendo. Se ponen así en funcionamiento heterotopías vinculadas a políticas sociales que a través de una función prestacional se dirigen a cada individuo en particular, identificado con nombre, apellido, número de documento y diagnóstico. Es decir, puedan describirse en su funcionamiento como políticas del individuo (Merklen, 2013).

El proceso de individualización que se hace visible en la época de las biopolíticas del encierro respecto de la patologización de cada fenómeno social que emerja, continúa presente como uno de los rasgos más específicos de estas políticas públicas, aunque ahora de un modo relativamente diferente. Las políticas públicas implican siempre una articulación de distintas tecnologías de poder, pero orientadas a objetivos concordantes con los que propone la gubernamentalidad vigente en cada época.

En este tipo de políticas públicas se requiere de una activación de parte de cada implicado. Y se trata de una activación que presenta dos facetas. Por un lado, activación de sí mismo para solicitar el acceso a cada territorialidad específica, con sus normas, sus circuitos, sus tiempos, etc. Y activación también porque estos mecanismos específicos destinados al abordaje de estas problemáticas sólo pueden ponerse en funcionamiento a partir de que un individuo lo solicita. Se encuentran disponibles para estos sectores de la población en tanto política pública, pero requieren de una activación que provenga del movimiento burocrático que cada individuo debe realizar por sí mismo para que ello se ponga en marcha.

Las políticas del individuo o políticas de individuación (Merklen, 2013) muestran una transformación en las relaciones entre el Estado y la sociedad, en tanto no funcionan en base a la socialización o la integración a la vida social, sino más bien a una "intervención sobre el otro" (Merklen, 2013:73). Se trata de un género de política pública que encuentra su blanco en el individuo mismo, siendo su propósito que éste se maneje de acuerdo a los principios de autonomía, requiriendo que sea responsable de sí mismo y que se mantenga activo en toda circunstancia. De esta manera, es posible que cada uno se asegure por sí mismo frente a los riesgos que la sociedad impone, haciéndose cargo de su propia experiencia vital y siendo el promotor de su propio proyecto de vida. 
Por ejemplo, en el territorio de la discapacidad mental, puede encontrarse que a partir de la legislación de 1981 (ley 22.431) se abre la posibilidad de una diagramación de estrategias generales para el sector poblacional de sujetos en situación de discapacidad, pero complementados a su vez por la exigencia de una conformación técnica de cada abordaje específico, de acuerdo a la singularidad de cada particularidad individual. La certificación de la discapacidad -otorgada por el Ministerio de Salud de cada provincia- viene a completar el esquema, funcionando como nexo entre una estrategia general (que intenta contemplar todas las posibilidades) y la necesidad de un abordaje singular (elaborado en función de las características particulares de cada beneficiario). La Convención sobre los Derechos de las Personas con Discapacidad y la ley de 2008 en la que Argentina adhiere a ella, consolidan el desplazamiento comenzando más de dos décadas antes, que va de un modelo de naturaleza rehabilitatoria y tutelar, hacia otro que apunta más bien a lo social y a la autonomía. "Hay medidas concebidas ahora sobre la base de ideas como el acompañamiento, la ayuda personalizada, la orientación, las formaciones, la proximidad, la participación” (Merklen, 2013:80).

De la misma manera, las políticas en torno a la niñez en situación de vulnerabilidad se ven transformadas desde una modalidad en la que priman los grandes espacios y el funcionamiento anónimo hacia políticas públicas definidas de acuerdo a cada caso en función de lo que los equipos interdisciplinarios de la Dirección de Niñez resuelvan. Así las cosas, el otrora hegemónico Hogar del Huérfano, con alrededor de 130 años de vigencia en la ciudad de Rosario, cede su lugar a una otras dos modalidades: Instituciones de Alojamiento Transitorio cuya capacidad es de no más de 30 niños y niñas, o bien individuos, parejas o familias que alojen los niños que deben ser retirados de su familia de origen para preservar sus derechos (ya sea una Familia Solidaria o el grupo familiar ampliado).

Ahora bien, esto conlleva una nueva dificultad: la invisibilización del hecho de que no todos los individuos se encuentran en las mismas condiciones subjetivas y políticas para responsabilizarse en llevar adelante los mecanismos necesarios destinados a enfrentar lo que su estado particular requiera. "Las clases populares sufren tanto los mandatos de individuación que los responsabilizan de su suerte como los cantos de sirena que les proponen mayor autonomía y movilidad cuando en realidad se encuentran despojadas de los recursos necesarios para enfrentarlas" (Merklen, 2013:56).

Por lo tanto, los procesos de inclusión sólo pueden devenir exitosos en los casos de individuos aislados que logren introducirse en los circuitos burocráticos que materializan las políticas de individuación. Pero nunca para todo un grupo de individuos, ni para una determinada región geográfica o una zona urbana específica, ni mucho menos para todo un sector poblacional. 
Se trata de una magnífica muestra de la forma en que la biopolítica y las anátomopolíticas, a través de las políticas del individuo, se articulan justamente para incidir en la regulación de determinadas problemáticas sociales, pero sin cuestionar ni afectar la modalidad de gubernamentalidad que las produce y las sostiene. En otras palabras:

Así, individuo y población se ensamblan en esas microscópicas mallas de las redes del poder mediante un despliegue de novedosas estrategias que operan con la mecánica de otras coerciones ocultas y sutiles. El control estadístico de todos los movimientos poblacionales (...) vehiculiza la regulación poblacional (Emmanuele \& Cappelletti, 2001:65).

La biopolítica de la inclusión respecto de los territorios de la discapacidad, la niñez vulnerada, las adicciones, el padecimiento mental, etc., exige cada vez más mecanismos y recursos, tanto legislativos como de políticas públicas, tendientes a contrarrestar la exclusión, la marginación, la opresión, la discriminación que denuncia este sector de la población. Sin embargo, esos esfuerzos que casi siempre se corresponden con la mejor de las intenciones, no reflejan en las estadísticas y en el vivenciar cotidiano de estos sectores poblacionales, mejoras acordes a dichos intentos. Es decir, podría sostenerse que esta clase de políticas públicas -caracterizadas por implicar una acción sobre un individuo concreto, identificado por nombre y apellido-, se presentan con la supuesta intención de solucionar ciertas injusticias sociales, pero lo máximo que pueden lograr es la reducción de su incidencia, regulando mínimamente la manifestación de tales problemas (Contino, 2013).

En conclusión, estas tácticas y estrategias tampoco deberían ser concebidas como formas incompletas o imperfectas de abordaje de la problemática, sino como la máxima modificación posible de ser realizada en la actualidad, sin conmover la racionalidad político-económica de las sociedades capitalistas. Por lo tanto, es esperable que nunca logren los objetivos propuestos, en tanto su accionar se encuentra limitado por una imposibilidad: toda biopolítica, siempre, debe dejar intacta la estructura político-económica de la gubernamentalidad actual. Aun cuando el costo de este límite sea no modificar significativamente la situación de quienes se encuentran vivenciando obstáculos inherentes a la problemática por la que se encuentran atravesados. 


\footnotetext{
${ }^{(*)}$ El presente trabajo se vincula a la tesis doctoral titulada "Institucionalización de la discapacidad intelectual en la ciudad de Rosario. Análisis de las políticas públicas, y de los procesos de subjetivación, autonomía e integración social", llevada a cabo en el marco de la carrera de Doctorado en Psicología de la Facultad de Psicología de la Universidad Nacional de Rosario y subsidiado mediante beca por el Consejo Nacional de Investigaciones Científicas y Técnicas (CONICET).

${ }^{1}$ Cabe destacar que el alienismo es la primera clínica médica sobre la locura y en tanto tal, puede ser concebido como el medio de legitimación social que el discurso médico encuentra para favorecer la modalidad de los espacios de encierro -Asilos- como modo hegemónico de abordaje.
}

\section{Referencias bibliográficas}

Albano, Sergio (2006) Arqueología del psicoanálisis. Una genealogía del discurso freudiano. Buenos Aires: Quadrata.

Belgich, Horacio (2004) Sujetos con capacidades diferentes. Sexualidad y subjetivación. Armenia: Kinesis.

Cohen, Hugo \& Natella, Graciela (Coords.) (2007) Trabajar en Salud Mental: la desmanicomialización en Río Negro. Buenos Aires: Lugar.

Contino, Alejandro (2010) "La especificidad de la función del psicólogo en el campo de la discapacidad mental". Revista de Psicología de la Universidad de Chile 19(2):157-182. http://www.revistapsicologia.uchile.cl/index.php/RDP/article/ viewPDFInterstitial/17114/17842 recuperado el 18.11.2013

(2013) “La estrategia de inclusión en discapacidad”. Revista Sujeto, Subjetividad y Cultura 5:59-66. Recuperado el 12.1.14 http://psicologia.uarcis.cl/revista/index. php/RSSCN1/article/view/110/95

Deleuze, Gilles (2005a) “Estado y máquina de guerra”. En C. Ferrer (Comp.). El lenguaje libertario (pp.167-195). La Plata:Terramar.

(2005b) "Postdata sobre las sociedades de control". En Christian Ferrer (Comp.). El lenguaje libertario. La Plata:Terramar, 2005:114-121.

Deleuze, Gilles \& Guattari, Felix (2002) Mil mesetas. Capitalismo y esquizofrenia. Valencia: Pre Textos. 
Droit, Roger-Paul (2008) "«Soy un artificiero». A propósito del método y la trayectoria de Michel Foucault”. En Entrevistas con Michel Foucault. Buenos Aires: Paidós, 2008:71-104.

Emmanuele, E. (1998) Educación, Salud, Discurso Pedagógico. Buenos Aires: Noveduc.

Emmanuele, Elsa \& Cappelletti, Andrés (2001) La vocación. Arqueología de un mito. Buenos Aires: Lugar.

Fernández Bruno, Mónica \& Gabay, Pablo (2003) Rehabilitación psiquiátrica y reinserción social de las personas con trastornos mentales crónicos y graves. Buenos Aires: Polemos.

Foucault, Michel (1998) Historia de la locura en la época clásica. México: Fondo de Cultura Económica.

(2004) Vigilar y castigar: nacimiento de la prisión. Buenos Aires: Siglo XXI.

(2008a) Los anormales: Curso en el Collège de France: 1974-1975. Buenos Aires: Fondo de Cultura Económica.

(2008b) El poder psiquiátrico: Curso en el Collège de France: 1973-1974. Buenos Aires, Fondo de Cultura Económica.

(2009) Seguridad, territorio, población: Curso en el Collège de France: 1977-1978. Buenos Aires: Fondo de Cultura Económica.

(2010) "Las heterotopías". En El cuerpo utópico. Las heterotopías. Buenos Aires: Nueva Visión, 2010:19-32.

Galende, Emiliano (2006) "Breve historia de la crueldad disciplinaria”. En Emiliano Galende \& Alfredo Kraut. El sufrimiento mental. El poder, la ley y los derechos Buenos Aires: Lugar, 2006:15-102.

Goffman, Erving (1970) Internados. Ensayos sobre la situación social de los enfermos mentales. Buenos Aires: Amorrortu.

Grossberg, Lawrence (2012) Estudios culturales en tiempos futuros: Cómo es el trabajo intelectual que requiere el mundo de hoy. Buenos Aires: Siglo XXI. 
Merklen, Denis (2013) “Las dinámicas contemporáneas de la individuación”. En Robert Castel et al. Individuación, precariedad, riesgos. ¿Desinstitucionalización del presente? Buenos Aires: Paidós, 2013:45-86.

Organización Mundial de la Salud (OMS) (2010) Salud mental y desarrollo: Poniendo el objetivo en las personas con problemas de salud mental como un grupo vulnerable. Recuperado el 25.11.13 de http://apps.who.int/iris/bitstream/10665/84757/1/9789962642657_spa.pdf 